\title{
A Low-Cost Millimeter-Wave Phase-Contrast Imaging Technique
}

\author{
Pierre Payet, Laurent Chusseau, and Jérémy Raoult \\ IES, Université de Montpellier, 860 rue de Saint Priest, 34095 Montpellier, France \\ Email: laurent.chusseau@umontpellier.fr
}

\begin{abstract}
In the framework of a near-field remote sensing imaging experiment we developed an interferometric detection scheme at the open end of the rectangular waveguide that serves as probe. To proceed with lock-in detection, the Gunn source is further commutated at $\mathrm{kHz}$ frequencies. This results not only in an intensity sensitive image of the sample but also in a phase-contrast image that bring complementary informations. We relate this new image formation process to Gunn nonlinearities occurring during the commutation process and plan to use it for near-field phase-sensing characterization at very low-cost.
\end{abstract}

\section{INTRODUCTION AND BACKGROUND}

$\mathbf{M}$ ICROWAVE reflectometry has been shown effective for studying the nature of materials dielectric and metal structures [1], [2]. This method has several advantages including being non-destructive, non-invasive, offer the possibility of low power with high-resolution and high-sensitivity imaging. An experiment was built at $60 \mathrm{GHz}$. The Gunn source feeds an interferometric detection scheme [3] whose measurement port is the open-end of a WR15 rectangular waveguide. The millimeter-wave source is connected to the port 3 of a magictee and then split in two equal parts at ports 1 and 2 that travel into the probe line and the reference line, respectively. The latter ends with a sliding short while the probe line is kept open and sensitive to any impedance modification in its vicinity. Output signal is measured by a Schottky detector at port 4 of the magic-tee and results of the interferometric recombination of these two signals. The Gunn source oscillator (Quinstar QTM-602001) is switched on and off at a frequency $f$ up to $10 \mathrm{kHz}$. The detection is obtained by a lock-in fed by the output signal with $f$ as reference.

\section{RESULTS}

We chose to apply this imagery technique to an encapsulated integrated circuit with aim to see chip details through the package. The microscopic view of a similar sample with package removed for further comparisons is given in Fig. 1a. One distinguish a centered rectangular chip that is connected to the pins of the square package via numerous gold wire bondings arranged horizontally and vertically. In the experimental arrangement, the $\mathbf{E}$-field component sensing the circuit is along the vertical axis.

The intensity map is given in Fig. 1b. One observes the most intense signal on the package pins. In the center of this red square, the chip position is foreseen and it is surrounded by (a)

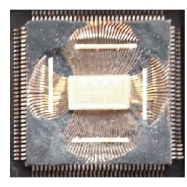

(b)

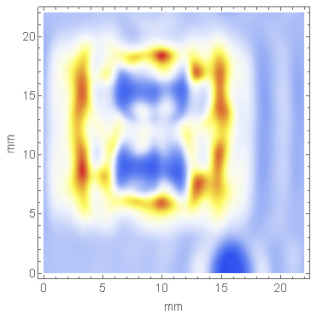

(c)

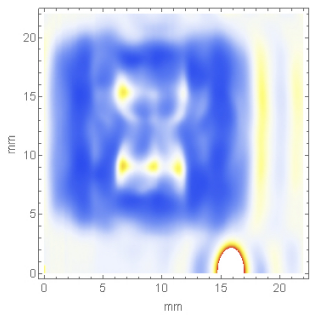

Fig. 1. (a) Circuit used as measurement sample with its package removed. (b) Intensity map of the sample. (c) Phase map of the sample.

the bonding appearing as a whole brighter mass. It should be notice that according to excitation polarization, only the horizontally oriented bonding sheets are visible.

The map of Fig. 1c is obtained simultaneously from the phase output of the lock-in. At first glance, it does not highlight the same things. Namely the package pins have been erased and most intense signals occur on the vertically oriented bonding wires. In fact a phase map is unexpected. A calculation of the detection process shows that both signals issued from the lock-in are proportional for an ideally switched source, i.e. the phase is a constant. Another physical effect operates here to produce the map of Fig. 1c, namely the switching of the Gunn diode induces a chirp that has been observed using a spectrum analyzer. If this chirp combines with frequency dependent properties of the sample, then the lock-in phase output is subject to variations that can be used for mapping. In our example the vertical bonding wires exhibit such a resonance and thus become the visible items.

To conclude, we take benefit from the non-ideal behavior of the Gunn source under switching to sense the dispersion of a sample in the mm-wave range. This low-cost technique, here used in the near-field, can be applied to any reflectometer and bring extra informations than just the intensity map.

\section{REFERENCES}

[1] M. T. Ghasr et al., "Comparison of near-field millimeter-wave probes for detecting corrosion precursor pitting under paint," IEEE Trans. Instrum. Meas., 54, pp. 1497-1504 (2005)

[2] S. Theerawisitpong et al., "In-depth measurement of $60 \mathrm{GHz}$ band nearfield and transmission mode microscopy," IRMMW-THz, Cardiff, UK, pp. 362-363 (2007)

[3] M. T. Ghasr et al., "Wideband millimeter wave interferometer for highresolution 3D SAR imaging," IEEE I2MTC, Pisa, IT, pp. 925-929 (2015) 